\title{
The EUSO-Balloon mission
}

\section{The JEM-EUSO Collaboration *}

EUSO-Balloon is a pathfinder for JEM-EUSO, the Extreme Universe Space Observatory which is to be hosted on-board the International Space Station. As JEM-EUSO is designed to observe Ultra-High Energy Cosmic Rays (UHECR)-induced Extensive Air Showers (EAS) by detecting their ultraviolet light tracks "from above", EUSO-Balloon is a nadir-pointing UV telescope too. With its Fresnel Optics and Photo-Detector Module, the instrument monitors a surface area of $\sim 50 \mathrm{~km}^{2}$ in a wavelength band of $290-430 \mathrm{~nm}$, collecting series of images at a rate of 400'000 frames/sec. The objectives of the balloon demonstrator are threefold : a) perform a full end-to-end test of a JEM-EUSO prototype consisting of all the main subsystems of the space experiment, $b$ ) measure the effective terrestrial UV background, with a spatial and temporal resolution relevant for JEM-EUSO. c) detect tracks of ultraviolet light "from above" for the first time. The latter is a milestone in the development of UHECR science, paving the way for any future space-based UHECR observatory. On August 25, 2014, EUSO-Balloon was launched from Timmins Stratospheric Balloon Base (Ontario, Canada) by the balloon division of the French Space Agency CNES. From a float altitude of $38 \mathrm{~km}$, the instrument operated during the entire astronomical night, observing UV-light from a variety of ground- covers, and simulated EAS, produced by flashers and a laser during a two-hour helicopter underflight. While a number of dedicated contributions in this volume focus on the results of the balloon flight and the performance of the EUSO-Balloon subsystems, this paper provides an overview of the instrument.

The 34th International Cosmic Ray Conference,

30 July- 6 August, 2015

The Hague, The Netherlands

*speaker: Petervon Ballmoos, IRAP Toulouse, France - E-mail: pvonballmoos@irap.omp.eu 


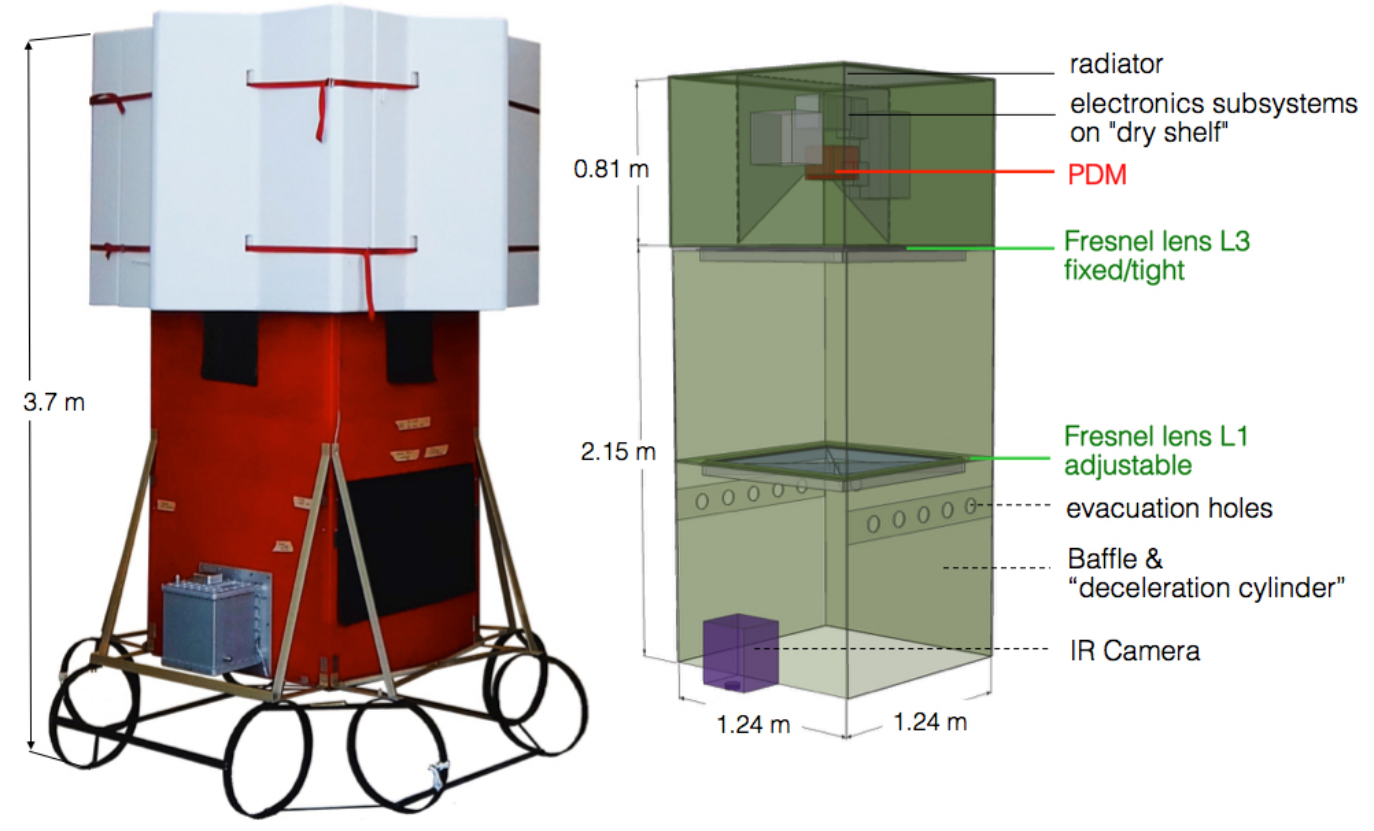

Figure 1: left: EUSO-Balloon, ready for its first flight, Timmins, Ontario (Ca), August 2014, right: schematic view of the instrument booth and optical bench, without floaters and "crash rings".

\section{Context}

EUSO-Balloon is developed by the JEM-EUSO collaboration as a demonstrator for the key technologies and methods featured in the forthcoming space instrument [1]. JEM-EUSO is designed to observe ultra high-energy cosmic rays (UHECRs) by looking downward to the Earth's atmosphere from the ISS, observing the UV fluorescence light of UHECR-induced Extensive Air Showers (EAS). This conference includes a number of detailed articles on JEM-EUSO, notably its status [1], the science case [2]; for an overview of the JEM-EUSO instruments see [3].

The EUSO-Balloon mission has been proposed in 2011 to the French Space Agency CNES by the three French laboratories (APC, IRAP and LAL) involved in the international JEM-EUSO collaboration. All relevant institutions and international partners within the JEM-EUSO collaboration contribute to the instrument (Table 1) according to their corresponding tasks and responsibilities within JEM-EUSO. As a CNES-led project, EUSO-Balloon was kicked off in September 2011 and went through a phase A study, demonstrating its feasibility by the end of the same year. The instrument definition (phase B) was carried out in 2012, with the production and the qualification of crucial subsystems (PDM, DP - see below) being led in parallel by the institutes of the JEM-EUSO collaboration. The manufacturing of all the subsystems was accomplished by the end of 2013, and the instrument was integrated and tested by June 2014. On August 25, 2014 EUSO-Balloon has performed a successful maiden flight after a launch by the French Space Agency CNES from the Stratospheric Balloon Base in Timmins, Ontario $(\mathrm{Ca})$. The present proceedings contain first results of this flight[4][5][6][7][8][9][10][11][12][13][14]. 


\section{The EUSO-Balloon instrument - overview}

The general layout of EUSO-Balloon is shown in Fig. 1, its main components are the optical bench and the instrument booth. Besides the focal plane detector (PDM) and associated electronics (DP) which are described below, the instrument booth houses the telemetry system (SIREN), CNES specific instrumentation (ICDV, Hub), and two battery-packs.

The Photo-Detector Module (PDM) The UV light collected by the telescope is focused onto and detected by - the PDM (Fig. 2), which is composed of 36 Multi-Anode Photomultiplier Tubes (MAPMT), associated front-end electronics, High-Voltage Power supplies, and trigger logic. The key characteristics of this UV camera are its spatial resolution of a few $\mathrm{mm}$, its time resolution of a few ns, a quantum efficiency of up to $30 \%$ making possible the detection of single photons, and its wide dynamic range over 6 orders of magnitudes. As the focal plane of JEM-EUSO will consist of 140 closely packed PDMs, the PDM of EUSO-BALLON has a modular design and is four-side buttable, i.e. its compact array of $6 \times 6$ MAPMT is covering a detection surface of $16.7 \times 16.7 \mathrm{~cm}^{2}$, and it is fully contained in a volume of $17 \times 17 \times 20 \mathrm{~cm}^{3}$ with its mechanical support and Front-End electronics included. The MAPMT's (Hamamatsu R11265-103-M64) have $8 \times 8$ pixels of $2.9 \times$ $2.9 \mathrm{~mm}^{2}$ each, and a global footprint of $26.2 \times 26.2 \mathrm{~mm}^{2}$. A UV color glass filter is bonded to the window of the MAPMT with optical glue. The filter (SCHOTT BG3 with anti-reflection coating) transmits UV light in a band between 290 and $430 \mathrm{~nm}$. At the rear of the MAPMT array, six ECASIC boards perform the full-time analog readout, detecting individual photons in each channel in order to count them per unit of time called the Gate Time Unit (1 GTU=2.5 $\mu \mathrm{s}$ ), performing the analog to digital conversion. The High-Voltage Power Supply (HVPS) consists of a miniaturized Cockroft-Walton (CW) generator [15], a switch system used to reduce the photocathode voltage to lower the MAPMT gain, and the logic system to manage the interfaces with the other elements of the electronic chain. Behind the EC-ASIC stack, the PDM board handles the interfaces between the various boards in the PDM and the Data Processor (6 EC-ASICs, HVPS in the PDM; the HK and CCB from the DP system - see below). Its core is an industrial Virtex6 XC6VLX240T FPGA. Besides managing the configuration, the PDM board governs the data stream from the 36 ASICs by means of the 6 EC-ASIC boards. The FPGA has to absorb a data-volume of 1 GBytes per second from the EC-Units, transmitting a part of the data to the Central Cluster Board (CCB) of the DP. The various parameters of the PDM's performance are treated in detail by a number of articles: photoelectron counting rate measurements [16]; sensitivity and detection performances [17]; absolute calibration [18].

The Data Processor (DP) The different sub-assemblies of the DP collect the PDM data, process them (trigger, time and position-tagging), handle their on-board storage, and send a subset to the telemetry system. The DP also includes the housekeeping system. The Control Cluster Board (CCB), developed around an FPGA Xilinx Virtex-4 FX-60, collects the data from the PDM board, processes and classifies the received data, and performs a second level trigger filtering. The Clock Board (CLKB) host the interface with the GPS receiver, it tags the events with their arrival time (UTC) and payload position (both provided by GPSR). It also measures the up-time and dead-time of the instrument and provides signals for time synchronization of the event. As the on-board triggers were not implemented on the 2014 flight, the CLK-Board also generated a "fake trigger 


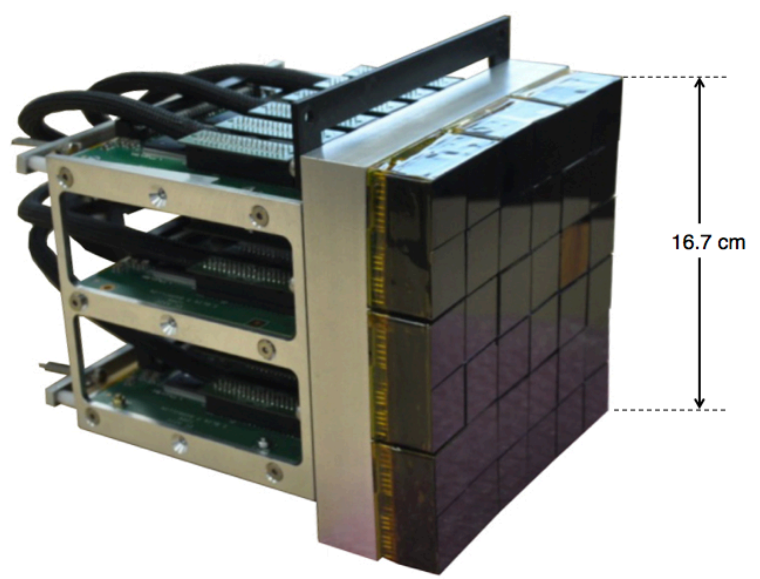

Figure 2: The Photo-Detector Module (PDM): the 36 Multi-Anode Photomultiplier Tubes (MAPMTs) are covered with UV filters; four MAPMTs form an Elementary Cell (EC). The PDM includes 9 ECs, 6 ECboards and a PDM board (see text).

signal", enabling the acquisition of events. The CPU, based on Atom N270 1.6 GHz processor, collects data from the CCB and CLKB through two (200 Mbits/sec) SpaceWire links. It manages the Mass Memory for data storage and handles the interface with the telecommand/telemetry system. One acquired event represents roughly $330 \mathrm{kB}$ of data. Since only a limited data rate can be transmitted to the ground through CNES' new NOSYCA telemetry system, all data are stored on board. The mass storage is composed of two Solid-State Drives (SSD), each one with 512 GB capacity operating in fault-tolerant mode RAID 1 disks (Redundant Array of Independent Disks). The House-Keeping (HK) system collects telemetry from several sub-systems of the instrument in slow control mode. It is responsible for monitoring voltages and currents of the Low Voltage Power Supply (LVPS), has a serial bus to convey telemetry and telecommands through the CPU interface and to other sub-systems. The HK is implemented around an off-the-shelf micro-controller board (Arduino Mega 2560), combined with 5 custom-made protocol interface boards to pre-process the various signals. The power is provided to the LV board and the HK board by two $28 \mathrm{~V}$ battery packs; the total power consumption of the electronics (DP and PDM) being $70 \mathrm{~W}$. A detailed description of the DP and its performance is given in [19].

The optics The optical bench contains two Fresnel lenses made of $8 \mathrm{~mm}$ thick PMMA (UV transmitting polymethyl-methacrylate) with a front surface of $100 \times 100 \mathrm{~cm}^{2}$ each. The EUSO-Balloon optics has been designed to resemble the JEM-EUSO optics: it is dimensioned to produce background rates per pixel comparable to the one anticipated for JEM-EUSO (i.e. roughly 1-1.5 photoelectrons per pixel in a $2.5 \mu \mathrm{sec}$ frame). The two lenses of the optical bench are shown in Fig. 3: L1 and L3 are aspherical Fresnel Lenses with focal lengths of $258.56 \mathrm{~cm}$ and $60.02 \mathrm{~cm}$, respectively (focal lengths are reference values only, single lenses are not producing stigmatic images). The position of L1 can be adjusted along the optical (Z) axis within the optical bench, the focal distance of the PDM is adjusted by a translation stage in the instrument boot. Together with the $16.7 \times 16.7 \mathrm{~cm}^{2}$ focal plane detector (PDM, see above) the optics provides a field of view of about $\pm 5.5^{\circ}$. A detailed description of the design and manufacturing of the balloon optics is given in [20] and [21]. The characterization of the fully integrated optical bench (global optical efficiency and point spread function) was performed at IRAP Toulouse and is reported in [22].

The Infrared Camera (IRcam) In order to monitor the cloud covers and particularly their height, the co-aligned IRcam observes the field of view of the main instrument. The general design of the 


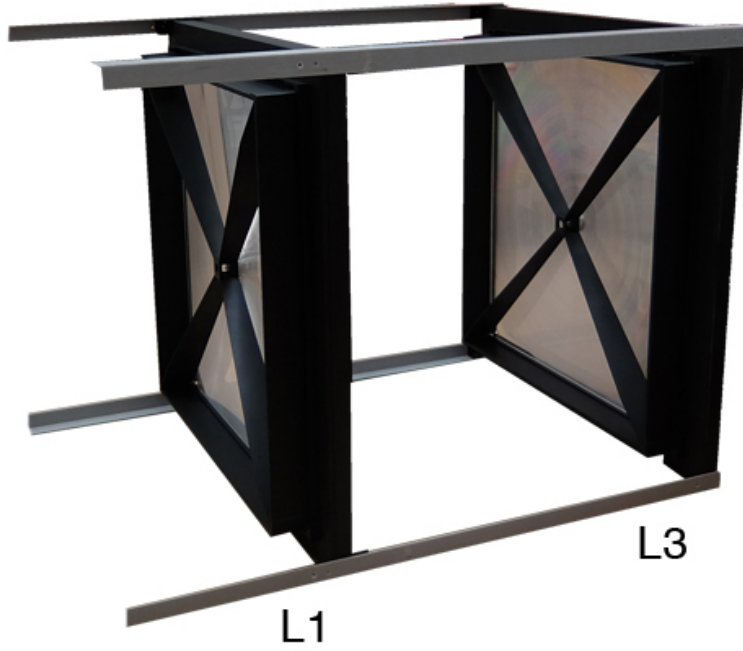

Figure 3: The Fresnel lenses L1 and L3 (8 mm thick PMMA, $1 \mathrm{~m}^{2}$ surface) mounted onto their fiberglass frames and spiders, and held at a distance of $1.11 \mathrm{~m}$ by an optical "sled".

IRcam is similar to the one used on JEM-EUSO. The camera provides images with a resolution of 640x480 pixels, in two wavelength bands centered at $10.8 \mu$ and $12 \mu$ (medium infrared), thanks to a ULIS UL 04171 microbolometer and two filters with $0.85 \mu \mathrm{m}$ bandwidth, centered on the above wavelengths. The data from the IRcam, along with auxiliary data (temperature, pressure and humidity), are stored in a RAID1 configuration of 2 SSD of 32 GB of capacity. The entire standalone IRcam system, including CPU and batteries, is housed in a rugged aluminium box $(0.4 \times 0.4$ $\times 0.4 \mathrm{~m}^{3}$ ) on the outside of the optical bench (see Fig. 1). A detailed description of the IRcam is presented in [24], its performance is detailed in [25].

The gondola The particular configuration of the nadir pointing instrument allowed to design a simple telescope structure serving simultaneously as a ballon gondola. The structure consists of two main modules, the optical bench and the instrument booth (see Fig. 1), both built from $10 \mathrm{~mm}$ Fibrelam aerospace panels assembled by Fribrolux L profiles. Fibrelam panels are manufactured from honeycomb that is bonded between composite facing skins; they are light-weight, structurally sound and exceptionally stiff. The dimensions of the fibrelam-telescope itself are $1.21 \times 1.21 \times$ $2.90 \mathrm{~m}^{3}$, the size of the flight-ready gondola is $2.58 \times 2.58 \times 3.7 \mathrm{~m}^{3}$, including "crash-rings" and floaters. The overall launch mass of the integrated instrument is $467 \mathrm{~kg}$.

Inside the self-contained, watertight instrument booth, all electronic equipment is mounted on a system of aluminum "shelves" that provides a thermal link to the $1.2 \times 1.2 \mathrm{~m}^{2}$ aluminum backplate (also called radiator). As the flight chain and harness interface to this backplate, it gets almost all the mechanical efforts at the opening shock of the parachutes. The entire gondola has been designed to withstand accelerations of up to $15 \mathrm{~g}$ along the $\mathrm{Z}$-axis, and $5 \mathrm{~g}$ in transverse $(\mathrm{X}, \mathrm{Y})$ directions. Besides its role as a structural element, the role of the radiator is to evacuate the excess energy generated by the electronics towards outer space.

Instead of "classic" crash-pads made of layered cardboard-honeycomb, EUSO-Balloon is using an ensemble of aluminium "crash-rings", designed to absorb the kinetic energy on ground impact through inelastic distortion (Fig 1). Due to the coincidence of a science requirement and a safety constraint, EUSO-Balloon has deliberately been designed to protect all sensitive equipment in the event of a water-landing. 
On its maiden flight in August 2014, EUSO-Balloon not only accomplished its science goals, but it also accidentally splashed down into a tiny solitary lake (barely bigger than two football fields) validating the water-landing capabilities it was designed for in the first place.

\section{The 2014 ballon flight from Timmins, Ontario}

On August 25, 2014 EUSO-Balloon was launched from the Timmins Stratospheric Balloon Base (lat $48.57 \mathrm{a}$ N, lon $81.38 \mathrm{~W}$ ). Thanks to the auxiliary balloon technique routinely used by CNES the entire launch-operations went very smoothly. The $467 \mathrm{~kg}$ payload was lifted from the airfield by a $400,000 \mathrm{~m}^{3}$ Zodiac balloon at 0:53 UT, reaching a float altitude of $38300 \mathrm{~m}$ at 3:43 UT (see Fig. 4 for a map with the flight path).

The moonless night of August 24/25 provided optimal conditions for the study of the UV background: a variety of ground covers have been overflown - including different types of soil and vegetation, wetlands, open water, urban and industrial areas, and various types of clouds. As it seemed unlikely to detect cosmic-ray induced air showers during this first short balloon flight, a pulsed UV laser and two UV flashers (LED and Xe) were shot into the field of view from a helicopter to simulate tracks of UV fluorescence photons, providing a means for the absolute calibration of the instrument [10]. The helicopter followed the balloon for over two hours, flying circles along the flight track at an altitude of $3000 \mathrm{~m}$ (Fig. 4).

Towards the end of the astronomical night at 8:20 UT, the flight was terminated (separation between the balloon and the instrument which descends on a parachute) about $100 \mathrm{~km}$ to the west of Timmins. Despite a descent path guaranteeing one of the "driest" landing zones along the flight track, EUSO-Balloon and the entire flight train splashed down in a small solitary lake at 8:59 UT. An adventurous recovery was performed by a crew of trappers, EUSO members, and with the help of a helicopter. Thanks to its inborn design for water-landings, the entire instrument was undamaged, both lenses were intact, the electronics, IRcam, and the RAID disks had not suffered any water damage and were fully operational. A general overview of the first results of the Timmins flight are summarized in [4] and references therein.

\section{Conclusions and Perspectives}

With its EUSO-Balloon flight in 2014, our collaboration has made a giant step towards demonstrating the key technologies and methods featured in JEM-EUSO. An ample part of the technological objectives have been achieved during this flight, the UV background was studied from a variety of ground covers, and the detection of hundreds of laser-shots produced during the helicopter underflight have demonstrated the ability for detecting of Extensive Air Showers from above. In order to achieve the ultimate objective of EUSO-Balloon, i.e. the first observation of UHECR induced Extensive Air Showers, the collaboration is planning two further balloon flights [26]. A first short flight will be carried out possibly as early as June 2016 with a launch from the CNES balloon-base in Aire-sur-l'Adour (France). The main goal is to test both an improved PDM and an achromatic optical bench, measure background above the Atlantic Ocean as well as simulated EASs (laser/flashers flown on an airplane), and validate strategies for the recovery of hardware and data in a planned splashdown at high sea. The second long or ultra-long duration balloon (ULDB) 


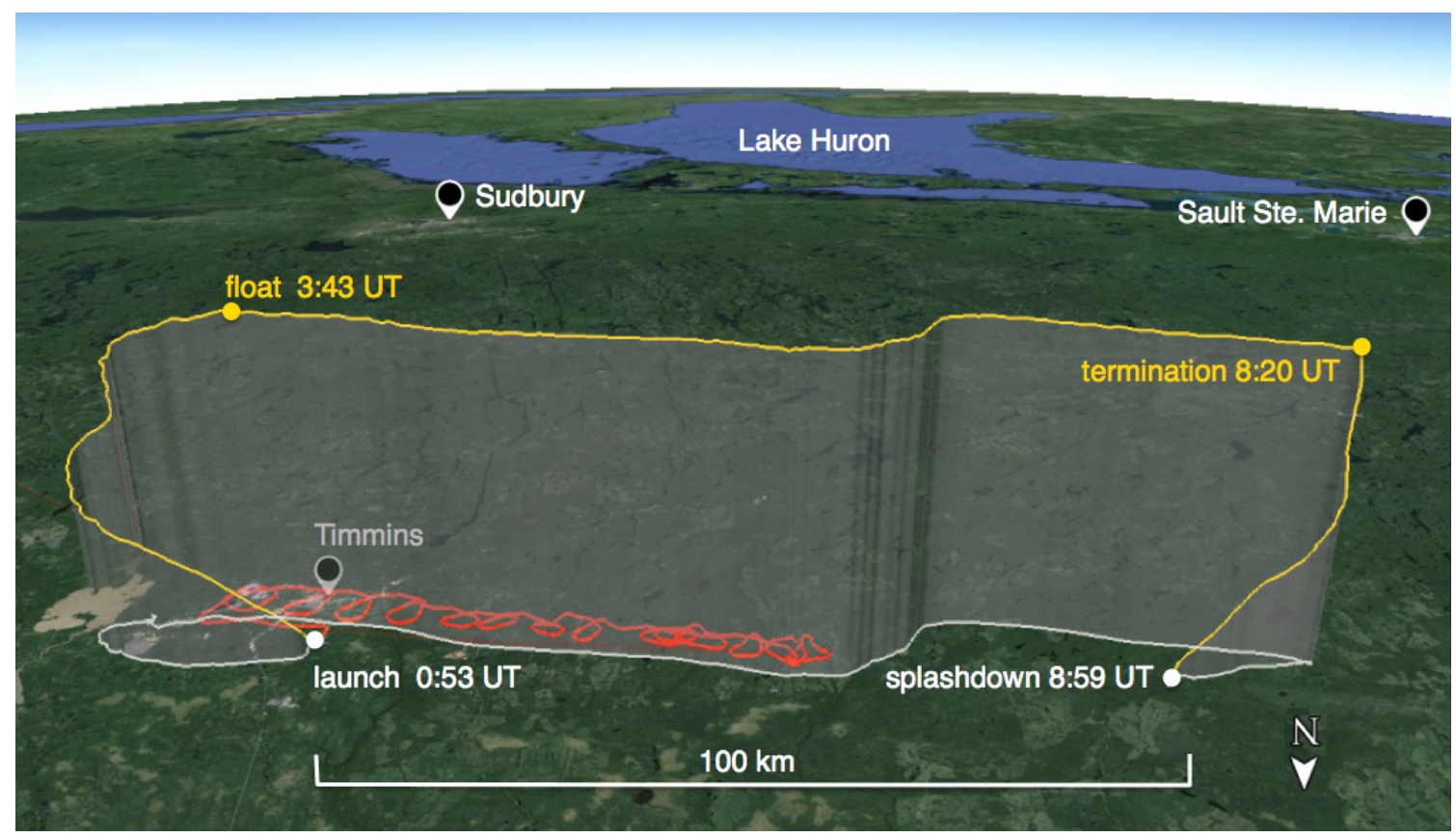

Figure 4: The flight-track of EUSO-Balloon on August 25, 2014 (yellow) - float altitude was $38 \mathrm{~km}$. The helicopter carrying the UV laser and two UV flashers followed the balloon for over two hours at an altitude of $3000 \mathrm{~m}$ (red).

flight shall achieve our ultimate goal, i.e. measuring a significant sample of UHECR induced tracks of UV light "from above". The flight could take place in spring 2017, most likely with a launch from the recently inaugurated base at Wanaka (NZ) by the Columbia Scientific Balloon Facility (CSBF/NASA). The trigger efficiency has been determined as a function of energy by [27], deriving the number of triggered EASs that can be expected during an ULDB flight in the range $10^{17}$ to $10^{19} \mathrm{eV}$. EUSO-Balloon has the potential to detect for the first time UV-light generated by atmospheric air-showers from near space. This milestone represents an important breakthrough in the development of UHECR science, paving the way for JEM-EUSO, and for any future space-based Cosmic Ray observatory.

Acknowledgment: The authors acknowledge strong support from the French Space Agency CNES who provided - besides funding - the leadership that made this achievement possible in a very short time. We are indebted to the balloon division of CNES for a perfect launch, smooth flight operation and flawless telemetry. The Canadian Space Agency has provided outstanding facilities at the Timmins Stratospheric Balloon Base, and a quick and careful recovery of the instrument. We would like to thank our laboratories and the entire JEM-EUSO collaboration for their strong and undivided support all along this project.

This work was partially supported by Basic Science Interdisciplinary Research Projects of RIKEN and JSPS KAKENHI Grant (22340063, 23340081, and 24244042), by the Italian Ministry of Foreign Affairs and International Cooperation, by the 'Helmholtz Alliance for Astroparticle Physics HAP' funded by the Initiative and Networking Fund of the Helmholtz Association, Germany, and by Slovak Academy of Sciences MVTS JEM-EUSO as well as VEGA grant agency project 2/0076/13. The Spanish consortium involved in the JEM-EUSO Space Mission is funded by MICINN under projects AYA2009- 06037-E/ESP, AYA-ESP 2010-19082, AYA2011-29489-C03- 01, AYA2012-39115-C03-01, CSD2009-00064 (Consolider MULTIDARK) and by Comunidad de Madrid (CAM) under project S2009/ESP-1496. 


\section{References}

[1] Santangelo, A., et al. (Proc. $34^{\text {th }}$ ICRC, 2015, The JEM-EUSO program

[2] Olinto, A., et al. Proc. $34^{\text {th }}$ ICRC, 2015, The JEM-EUSO science

[3] Casolino, M. et al, An overview of the JEM-EUSO instrument, Exp. Astr. (2014)

[4] Bertaina, M., et al., Proc. $34^{\text {th }}$ ICRC, 2015, Preliminary results from the EUSO-Balloon flight

[5] Eser, J., et al., Proc. 33 ${ }^{\text {rd }}$ ICRC, 2015, Laser direction reconstruction in EUSO-Balloon experiment

[6] Mackovjak, S.,34 ${ }^{\text {th }}$ et al., Proc. $34^{\text {th }}$ ICRC, 2015, Night time measurement of the UV background by EUSO-Balloon

[7] Jung, J., et al., Proc. $34^{\text {th }}$ ICRC, 2015, Search for significant background fluctuations in the EUSO-Balloon data

[8] Suino, G., et al., Proc. $34^{\text {th }}$ ICRC, 2015, Tests of JEM-EUSO $1^{\text {st }}$ level trigger using EUSO-Balloon data

[9] Fenu, F., et al., Proc. $34^{\text {th }}$ ICRC, 2015, The Simulation of cosmic rays in EUSO-Balloon: performance in the direction and energy reconstruction

[10] Adams, J., et al., Proc. $34^{\text {th }}$ ICRC, 2015, The Calibration of EUSO-Balloon using airborne light sources mounted to a Helicopter

[11] Panico, B., et al., Proc. $34^{\text {th }}$ ICRC, 2015, Analysis of EUSO-Balloon data with Offline

[12] Saez, G., et al., Proc. $34^{\text {th }}$ ICRC, 2015, Cloud Optical Depth obtained from the IR Camera data and the UV Flashers flying under the EUSO-Balloon

[13] Merino, A., et al., Proc. $34^{\text {th }}$ ICRC, 2015, Cloud top height estimation from WRF model: application to the infrared camera onboard EUSO-Balloon

[14] Tabone, I., et al., Proc. $34^{\text {th }}$ ICRC, 2015, The WRF model contribution to the atmospheric conditions estimation during the EUSO-Balloon experiment

[15] Blaksley, C., et al., Proc. $34^{\text {th }}$ ICRC, 2015, A Cockcroft-Walton High-Voltage Power Supply for the EUSO Instruments

[16] Rabanal, J., et al., Proc. $34^{\text {th }}$ ICRC, 2015, Photoelectron counting rate measurements in the UV camera during the EUSO-BALLOON night flight

[17] Dagoret, S., et al., Proc. $34^{\text {th }}$ ICRC, 2015, Determination of the sensitivity and detection performances of the UV camera pixels of the EUSO-BALLOON instrument

[18] Moretto, C., et al., Proc. $34^{\text {th }}$ ICRC, 2015, Absolute calibration of the photon detector module of the EUSO-Balloon experiment and improvements for future missions

[19] Osteria, G. et al., Proc. $34^{\text {th }}$ ICRC, 2015, The Data Processor System of EUSO-Balloon: in flight performance

[20] Takizawa, Y., et al., Proc. 33 ${ }^{\text {rd }}$ ICRC, 2013 (ID832), The TA-EUSO and EUSO-Balloon optics designs

[21] Takizawa, Y., et al., Proc. $33^{\text {rd }}$ ICRC, 2013 (ID1040), Manufacturing of the TA-EUSO and the EUSO-Balloon lenses

[22] Catalano, C. et al., Proc. $34^{\text {th }}$ ICRC, 2015, Performance of the EUSO-Balloon optics

[23] Morales et al., J.A., Proc. 33 ${ }^{\text {rd }}$ ICRC, 2013 (ID514), An End to End Simulation code for the IR-Camera of the JEM-EUSO Space Observatory

[24] Rodriguez Firas, M.D., et al., Proc. $34^{\text {th }}$ ICRC, 2015, The Infrared Camera on EUSO-Balloon

[25] Fernandez, J., et al., Proc. $34^{\text {th }}$ ICRC, 2015, Performances of the Infrared Camera on EUSO-Balloon

[26] Wiencke, L., et al., Proc. $34^{\text {th }}$ ICRC, 2015, EUSO-Balloon mission to record extensive air showers from near space

[27] Bacholle, S., et al., Proc. 34 ${ }^{\text {th }}$ ICRC, 2015,EUSO-Balloon trigger efficiency in preparation of a long duration flight 